JASC 12-1-3

\title{
Fairness CSMA/CA MAC Protocol for VLC Networks
}

\author{
Vu Van Huynh, Yeong Min Jang ${ }^{\dagger}$ \\ Department of Electronics Engineering, Kookmin University, Seoul, Korea
}

\begin{abstract}
This paper presents a fair MAC protocol based on the CSMA/CA algorithm in visible light communication (VLC) networks. The problem of bandwidth sharing among differentiated priority in VLC networks can be solved by using number of backoff time and backoff exponent parameters with AIFS. The proposed algorithm can achieve fair allocation of the bandwidth resource among differentiated priority. The two dimension Markov chain is assisted for analyzing the proposed mechanism about throughput and delay metrics. Numerical results show that our proposed algorithm improves the fairness among different traffic flows.
\end{abstract}

Key words : VPAN; CSMA/CA; fair MAC; Markov chain; AIFS.

\section{INTRODUCTION}

In recent years, Visible light communication (VLC) is one type of short-range optical wireless communication system in which visible light is used as a transmission medium [1]. When compare with radio frequency system, VLC has some advantages such as: harmless to human body, providing high security, high data rates, license free frequency band, and no interference with radio frequency system (RF) especially aircraft equipment or medical instruments. Some schemes to support fairness had been proposed in literatures [2]-[6]. In paper [2], a fair scheme which uses differentiations of IFS (inter frame space) and $\mathrm{CW}$ (contention window) for two classes of STA (stations) is presented in an 802.11e WLAN. The service index-based fairness scheduling algorithm (SIB-FS) which accounts for the service received by each flow and adjusts the backoff time for fair service is presented in [3]. Albert Banchs and Xavier Perez [4] extended the DCF (distributed coordinator function) of IEEE 802.11 to provide weighted fair queuing in WLAN. A fair MAC protocol for IEEE 802.11 based ad hoc networks was proposed in [5] to design practical fair media access control frameworks. Paper [6] proposed a priority-based fair scheduling algorithm for subscriber stations to serve a mixture of uplink traffic from different scheduling services. There are many literatures

Manuscript received Jan. 26, 2012; revised Feb.23, 2012

†Corresponding Author: yjang@kookmin.ac.kr

Tel: +82-2-910-5068, Fax: +82-2-910-5068, Kookmin Univ.

Dept. of Electronics Engineering, Kookmin Univ., Korea which have analyzed the CSMA/CA algorithm by using a discrete-time Markov chain model [7]-[10]. In this paper, we proposed a modified CSMA/CA algorithm to support fair MAC protocol for differentiated priority. Our mechanism uses backoff exponent (BE), number of backoff times (NB) and arbitration inter frame space (AIFS) to control the channel access procedure.

The rest of this paper is organized as follows: The proposed fair MAC protocol based on CSMA/CA algorithm is described in Section 2; Analytical model and performance analysis is addressed in Section 3; Numerical results are given in Section 4; finally Section 5 concludes this paper.

\section{Proposed a FAIR MAC PRotocol baSEd CSMA/CA}

In this subsection, we describe the operation of modified CSMA/CA algorithm of IEEE 802.15.7 to provide fair protocol among differentiated priority in Fig. 1. The CSMA/CA algorithm first initializes the NB to zero and two values $B E$ and $N B_{\max }$ depend on the priority levels of packet. Based on $B E$ value, the algorithm locates the boundary of the next backoff period for the device. The device generates a random number in the range 0 to $\left(2^{B E[l]}-1\right)$ and then it waits until the random number is reduced to zero in order to perform CCA. The PHY layer performs a CCA to check whether the channel is busy or not. If the channel is sensed busy, the algorithm will increase the $N B$ and $B E$ values by. If $N B$ exceeds the $N B_{\max }$ value, then the packet is discarded due to channel access failure. If the $N B$ value is equal or less 
than $N B_{\max }$ value, the algorithm will relocate backoff period boundary. Then it will apply backoff delay process again. If the channel is idle, the packet is transmitted successfully.

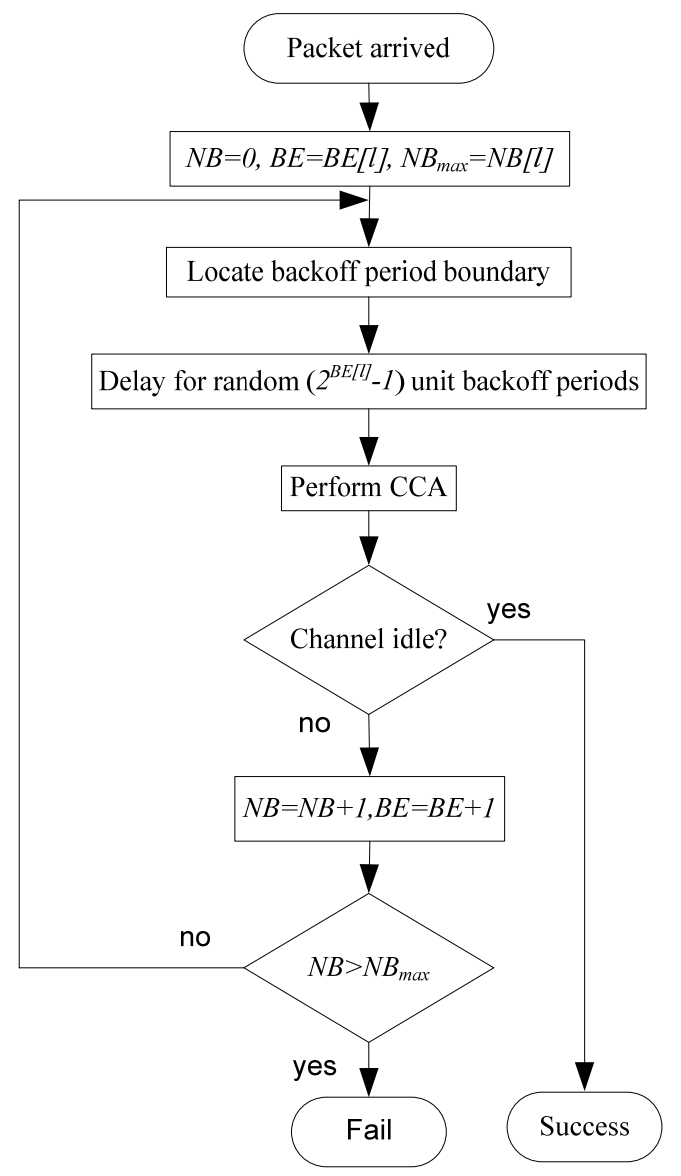

Fig. 1. CSMA/CA MAC protocol for supporting fairness

\section{ANAlytical Model}

Let $s(l, t)$ and $c(l, t$, be the stochastic processes values representing the value of $N B$, value of the backoff counter $(B E)$ at time t, respectively. Fig. 2 represents the Markov chain model which a node transmits a packet. In Fig. 2, we denote some MAC parameters such as: $W_{0}=2^{B E[l]}, W_{i}=$ $2^{i} W_{0}, m[l]=N B_{\max }=m$ for packet with priority $l$ level. $(-1,0)$ represents the state of an empty queue of a device. $\alpha$ is the probability that the channel is busy when a device in $l$ level is sensing in CCA procedure. $P_{0}$ is the probability that the queue has packet to send.

$$
\begin{gathered}
P\{-1,0 \mid-1,0\}=1-P_{0} \\
P\{0, j, \mid-1,0\}=P_{0} / W_{0}, j \in\left[0, W_{0}-1\right] \\
P\{i, j-1 \mid i, j\}=1, i \in[0, m], j \in\left[1, W_{i}-1\right] \\
P\{-1,0 \mid i, 0\}=\left(1-P_{1}\right)(1-\alpha), i \in[0, m] \\
P\{-1,0 \mid m, 0\}=P_{1} \alpha
\end{gathered}
$$

Equation (1) is the probability of empty queue which was empty in the previous state. Equation (2) shows the probability of going back to the first backoff stage from the idle stage. Equation (3) represents the reducing of backoff counter. Equation (4) represents the probability of going back to the empty queue stage after packet transmission. Equation (5) represents the probability of going back to the idle stage due to the retry limit.

We have the stationary distribution of the Markov chain model as: $b_{i, j, k}=\lim _{t \rightarrow \infty} P\left\{s(l, t)=i, c(l, t)=j, r(l, t)=k_{j}, i \in[0\right.$, $m], j \in\left[-C W, W_{i}-1\right], k \in[0, n]$. With the special case $\mathrm{b}_{-1,0,0}$ $=\lim P(-1,0,0)$.

By the normalization condition, we know that:

$$
b_{-1,0}+\sum_{i=0}^{m} \sum_{j=0}^{W_{i}-1} b_{i, j}=1
$$

According to the relationship between stable states we have:

$$
\begin{gathered}
b_{i, 0}=\alpha b_{i-1,0} \\
b_{i, 0}=\alpha^{i} b_{0,0}
\end{gathered}
$$

With the Markov chain regularity we have:

$$
\begin{aligned}
& b_{i, j}=\frac{W_{i}-j}{W_{i}} b_{i, 0} \\
& b_{-1,0}=\frac{1-P_{1}}{P_{0}} b_{0,0}
\end{aligned}
$$

Based on the above formulas, we calculate apart of equation (6):

$$
\begin{aligned}
\sum_{i=0}^{m} \sum_{j=0}^{W_{i}-1} b_{i, j} & =\sum_{i=0}^{m} \sum_{j=0}^{W_{i}-1} \frac{W_{i}-j_{i}}{W_{i}} b_{i, 0}=\sum_{i=0}^{m} \frac{W_{1}+1}{2} \alpha^{i} b_{0,0} \\
& =\frac{\left[\left(2^{m+1}-1\right) W_{0}+1\right]\left(1-\alpha^{m+1}\right)}{2(1-\alpha)} b_{0,0}
\end{aligned}
$$

Substituting equation (10) and equation (11) into equation (6) we have:

$$
\begin{aligned}
& \frac{1-P_{1}}{P_{0}} b_{0,0}+\frac{\left[\left(2^{m+1}-1\right) W_{0}+1\right]\left(1-\alpha^{m+1}\right)}{2(1-\alpha)} b_{0,0}=1 \\
& b_{0,0}=\frac{2 P_{0}(1-\alpha)}{2\left(1-P_{1}\right)(1-\alpha)+P_{0}\left(1-\alpha^{m+1}\right)\left[W_{0}\left(2^{m+1}-1\right)+1\right]}
\end{aligned}
$$

The probability that a device in priority $l$ level tries to transmit packet in the unit backoff period:

$$
\omega_{l}=b_{-1,0}+\sum_{i=0}^{m} b_{i, 0}=\frac{1-P_{1}}{P_{0}} b_{0,0}+\sum_{i=0}^{m} \alpha^{i} b_{0,0}
$$




$$
=\frac{\left(1-P_{1}\right)(1-\alpha)+P_{0}\left(1-\alpha^{m+1}\right)}{P_{0}(1-\alpha)}
$$

Based on $\omega_{l}$ value, we will calculate $\alpha$ :

$$
\alpha=1-\sum_{i=0, i \neq l}^{L}\left(1-\omega_{i}\right)^{n_{i}}\left(1-\omega_{l}\right)^{n_{l}-1}=1-\frac{1}{1-\omega_{l}} \prod_{i=0}^{L}\left(1-\omega_{i}\right)^{n_{i}}
$$

Let $\mathrm{S}[1]$ be the normalized throughput of the device in the 1 priority level, with $1 \ni[0, \mathrm{~L}]$. Then the normalized throughput can be expressed as the following ratio:

$$
\begin{gathered}
S[1]=\frac{E[\text { average successful transmitted data bytes] }}{E[\text { average time inteval }]} \\
=\frac{P_{s}[1] \mathrm{T}_{p}}{P_{I} \delta+P_{s} T_{s}+P_{c} T_{c}}
\end{gathered}
$$

where $P_{s}, P_{I}, P_{c}, T_{p}, T_{s}$, and $T_{c}$ are the probability of a successful transmission, idle channel, busy channel, the time to transmit the packet payload, the average durations for successful transmission and collision, respectively.

We define the delay of a packet, $D$, which is the time elapsed from the instant of the generation of the packet to the instant of the successful reception or drop of it. The value of $D$ is depended on the $l$ priority level. So, $E(D)$ is the mean value of $D$. The mean value $E(D)$ can be given by:

$$
E(D[l])=E\left(N_{c}[l]\right)\left[E(B[l])+T_{c}\right]+\left(E(B[l])+T_{s}\right)
$$

where $N_{c}[l]$ is the number of collision before transmitting a packet for the priority $l$ level. $B[l]$ denotes the time interval of average backoff delay and the CCA delay. So $E([l])$ is the mean value of $B[l]$.

\section{NUMerical Results}

In this section, the modified CSMA/CA algorithm with AIFS will be validated through two performance metrics: throughput and transmission delay. The numerical result also bases on the parameters in specifications of the IEEE 802.15.7 in [1]. We used the PHY type I with OOK modulation scheme and $24.4 \mathrm{~kb} / \mathrm{s}$ data rate in the numerical performance.

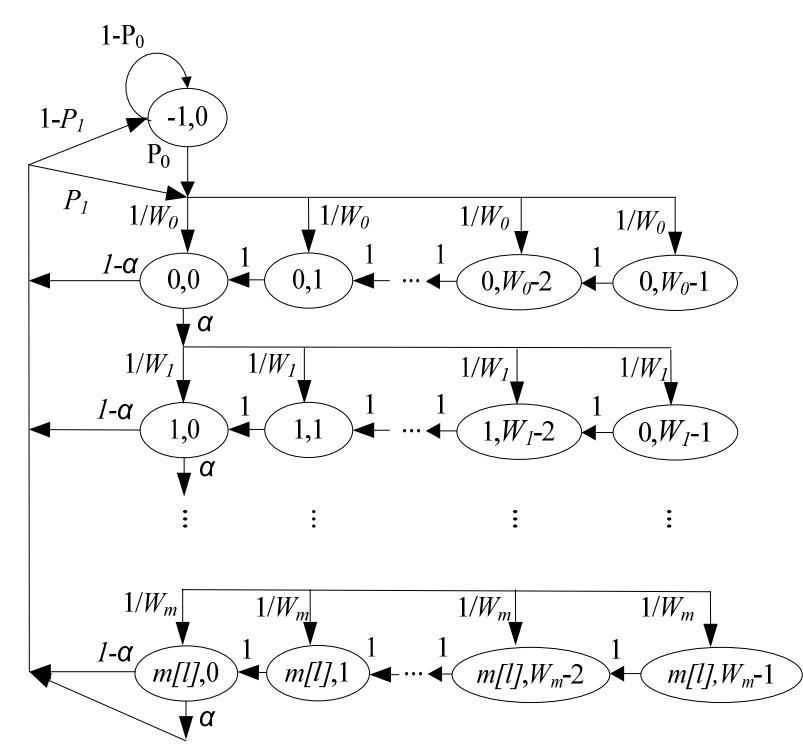

Fig. 2. Markov Chain model.

We also assume that the numbers of devices in each priority level are same and the maximum numbers of devices in each level are 10 devices. We set $L=1$ in the numerical results or there are two priority levels. So, we have high priority $(L=0)$ and low priority $(L=1)$. Each priority class is assigned an AIFS. To investigate the impact of multi-parameters on the VPAN, we set the multi-parameters correlative with high priority and low priority as follow: $B E[l]$ are set to 3 and 5, respectively, and $N B[l]$ is set to 4 for any $B E[l]$ value. $N B[l]$ are set to 6 and 2 , respectively, and $B E[l]$ is set to 4 for any $N B[l]$ value.

In Fig. 3 and Fig. 4, we reported the throughput and transmission delay when the number of devices increases with proposed fair MAC protocol in analysis. The throughput is decreased as number of devices is increased because of sharing the bandwidth capacity. The transmission delay is increased as number of devices is increased due to the probability of a collision becomes larger. The higher priority achieves higher throughput and lower transmission delay. 


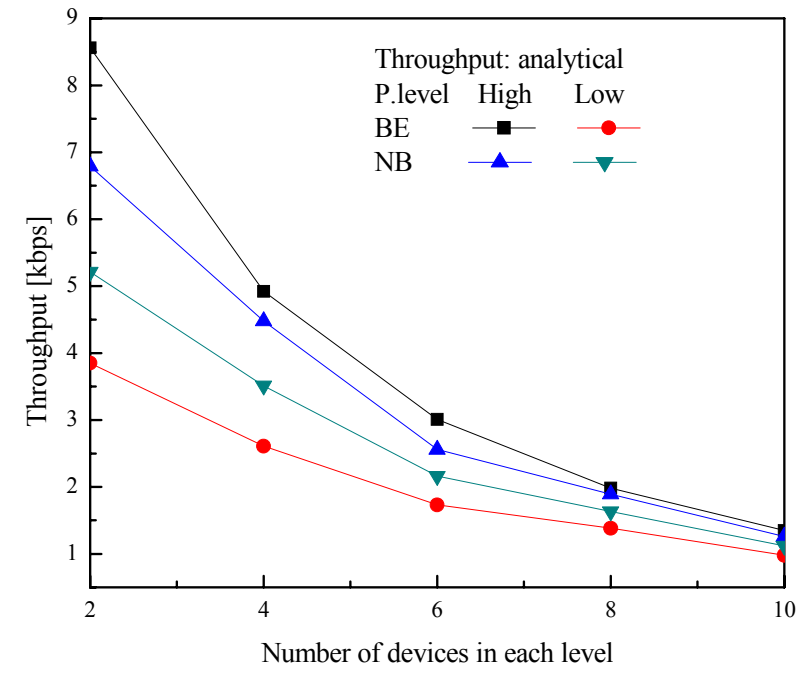

Fig. 3. Comparison of throughput with different levels.

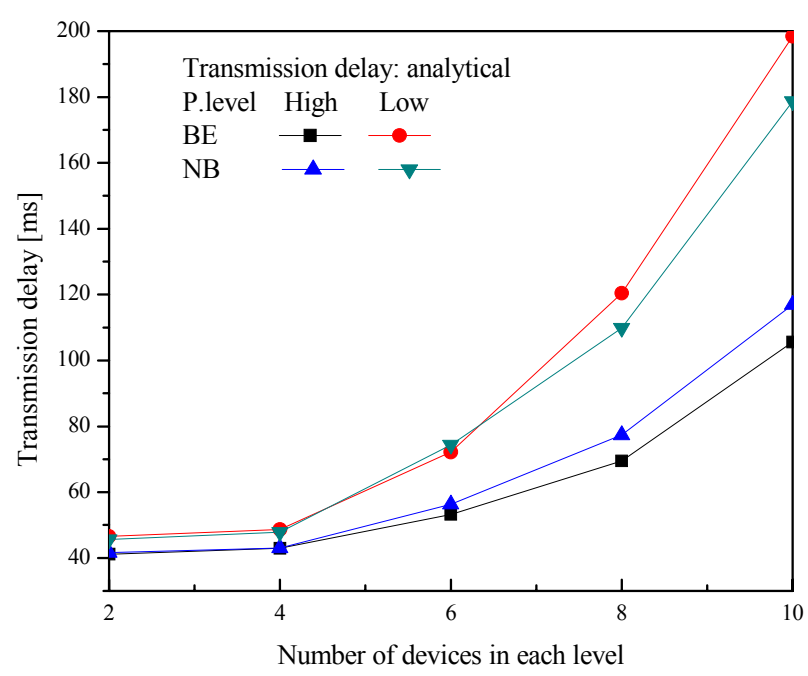

Fig. 4. Comparison of transmission delay with different levels.

\section{CONCLUSION}

This work presents a fair MAC protocol using modified CSMA/CA algorithm with AIFS in IEEE 802.15.7 VLC system. A discrete time Markov is used to model the procedure of proposed mechanism and analyze the throughput and transmission delay. The numerical results show that our proposed mechanism is appropriate to support fair MAC protocol for differentiated priority.

\section{ACKNOWLEDGMENT}

This work has been supported partially by 'Broadcasting-Communications R\&D Program' of Korea Communications Commission/Korea Communications Agency (No. 11911-01111).

\section{REFERENCES}

[1] IEEE P802.15.7, Part 15.7: PHY and MAC Standard for Short-range Wireless Optical Communication Using Visible Light; Available online: http://ieeexplore.ieee.org/xpls/abs_all.jsp?arnumber=601 6195 (accessed on 20 March 2012).

[2] R. G. Cheng, C. J. Chang, C. Y. Shih, and Y. S. Chen, "A New Scheme to Achieve Weighted Fairness for WLAN Supporting Multimedia Service," IEEE Trans. Wireless Communications, Vol. 5, pp. 1095-1102, 2006.

[3] Y. Q. Chen, K. M. Roh, and S. J. Yoo, "Service Index-based Fairness Scheduling in Wireless Ad Hoc Networks," Computer Communications, Elsevier., Vol. 29, pp. 2934-2944, 2006.

[4] A. Banchs and X. Perez, "Distributed Weighted Fair Queuing in 802.11 Wireless LAN," in Proc. ICC communications of IEEE international conference, pp. 3121-3127, 2002.

[5] B. Bensaou and Z. Fang, "A Fair MAC Protocol for IEEE 802.11-Based Ad Hoc Network: Design and Implementation," IEEE Trans. Wireless Comm., Vol. 6, pp. 2934-2941, 2007.

[6] Y. Wang, S. Chan, M. Zukerman, and R. J. Harris, "Priority-based Fair Scheduling for Multimedia WiMAX Uplink Traffic," IEEE ICC, pp. 301-305, 2008.

[7] M. E. Rivero-Ángeles, D. Lara-Rodríguez, and F. Cruz-Pérez, "Differentiated Backoff Strategies for Prioritized Random Access Delay in Multiservice Cellular Networks," IEEE Trans. Vehicular Tech. Vol. 58, pp. $381-397,2009$.

[8] J. He, L. Zheng, Z. Yang, C. T. Chou, and Z. Tang, "Analytical Model for Service Differentiation Schemes for IEEE 802.11 Wireless LAN," IEICE Trans. Communications, Vol. E87-B, pp. 1724-1729, 2004.

[9] J. Misic, S. Shafi, and V. B. Misic, "Performance of a Beacon Enabled IEEE 802.15.4 Cluster with Downlink and Uplink Traffic," IEEE Trans. Parallel Distributed System, Vol. 17, pp. 361-376, 2006.

[10] V. V. Huynh, L. N. Tuan, and Y. M. Jang, "Priority MAC based on Multi-parameters for IEEE 802.15.7 VLC in Non-saturation Environment," Journal of Korea Information and Communications Society (KICS), Vol. 37C, pp. 224-232, 2012. 


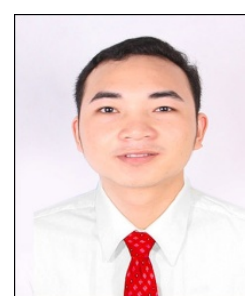

\section{Vu Van Huynh}

He received the diploma in Faculty of information and communication Technology from Ha Noi University of Science and Technology, Viet Nam in 2010. He is currently Master student in Department Electronics Engineering, Kookmin University, Korea. His research interests are visible light communication, MAC protocol, RFID, LED-ID, and localization based service.

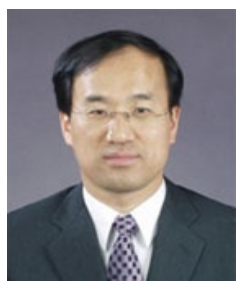

\section{Yeong Min Jang}

He received the B.E. and M.E. degree in Electronics Engineering from Kyungpook National University, Korea, in 1985 and 1987, respectively. He received the doctoral degree in Computer Science from the University of Massachusetts, USA, in 1999. He worked for ETRI between 1987 and 2000. Since September 2002, he is with the School of Electrical Engineering, Kookmin University, Korea. His research interests are $5 \mathrm{G}$ Mobile, radio resource management, LED-ID, VLC networks, multi-screen service, and convergence networks. 